\author{
F. Piazza $\cdot$ P. De Los Rios $\cdot$ D. Fanelli \\ L. Bongini · U. Skoglund
}

\title{
Anticooperativity in diffusion-controlled reactions with pairs of anisotropic domains: a model for the antigen-antibody encounter
}

\begin{abstract}
The encounter between anisotropic agents in diffusion-controlled reactions is a topic of very general relevance in chemistry and biology. Here we introduce a simplified model of encounter of an isotropic molecule with a pair of partially reacting agents and apply it to the encounter reaction between an antibody and its antigen. We reduce the problem to the solution of dual series relations, which can be solved iteratively, yielding the exact solution for the encounter rate constant at any desired order of accuracy. We quantify the encounter effectiveness by means of a simple indicator and show that the two binding centers systematically behave in an anticooperative fashion. However, we demonstrate that a reduction of the binding active sites allows the composite molecule to recover binding effectiveness, in spite of the overall reduction of the rate constant. In addition, we provide a simple formula that enables one to calculate the anticooperativity as a function of the size of the binding site for any values of the separation between the two active lobes and of the antigen size. Finally, some biological implications of our results are discussed.
\end{abstract}

Keywords Diffusion-controlled reactions - Encounter rate constant - Anisotropic reactants .

Anticooperativity $\cdot$ Antibodies

F. Piazza $(\bowtie) \cdot$ P. D. L. Rios

Laboratoire de Biophysique Statistique,

SB-ITP, Ecole Politechnique Fédérale de Lausanne, BSP-720, 1015 Lausanne, Switzerland

E-mail: francesco.piazza@epfl.ch

D. Fanelli

Dipartimento di Energetica "S. Stecco", Università di Firenze, Via S. Marta 3, 50139 Florence, Italy

D. Fanelli · L. Bongini · U. Skoglund

Department of Cell and Molecular Biology,

Karolinska Institutet, 17177 Stockholm, Sweden

\section{Introduction}

The study of reactions and binding between anisotropic macromolecules is an issue at the core of molecular biology. Among the former processes, the binding between foreign agents of different nature (antigens) and antibodies in higher organisms is an example of the utmost relevance. The unexpected presence of intruder organisms, such as viruses or bacteria, may trigger fast immunological responses of a different kind, by rapidly letting B cells develop high concentrations of antibodies of the relevant family in the specific tissue or in the blood.

Antibodies are large molecules of about $150 \mathrm{kDa}$. Their structure, common to all families, consists of three large domains, joined in the central, flexible region of the molecule and relatively free to move with respect to each other. In Fig. 1 we show a threedimensional reconstruction at $2-\mathrm{nm}$ resolution of a murine antibody immunoglobulin $\mathrm{G}$ ( $\mathrm{IgG}$ ) by means of cryo-electron tomography (Sandin et al. 2004). The two identical upper large lobes are known as antigenbinding (Fab) arms, while the lower, slightly flatter structure is known as the Fc fragment (so called because it is readily crystallized). The tips of the Fab fragments contain the active sites: these are the loci where the binding with a given antigen takes place. The amino acid sequence of the two identical active regions is hypervariable, and is the result of a complex process involving both somatic recombination and selective gene splicing during B-cell differentiation (Voet and Voet 1995). This allows a single organism to have at its disposal a huge variety of antibody molecules which are almost identical regarding their structure and dynamics, while targeting an enormous number of different external agents.

The most general mechanism of antigen-antibody binding can be represented as a two-step reaction of the kind 


$$
\begin{aligned}
\text { Antigen }+ \text { antibody } & \underset{\kappa_{-1}}{\stackrel{\kappa}{\rightleftarrows}} \text { encounter complex } \\
& \underset{\kappa_{-2}}{\stackrel{\kappa_{2}}{\rightleftarrows}} \text { complex. }
\end{aligned}
$$

The first reversible reaction describes the encounter between an antigen and an antibody in vivo. This process is driven by diffusion. Interestingly, it has recently been proved that the dynamics of large-scale motions of the antibody plays a crucial role in determining the encounter rate $\kappa$ (Bongini et al. 2004). The following stage of the reaction describes the chemical fixation of the antigen-antibody complex. That is, for example, where the (unknown) chemical binding affinity enters play.

The calculation of the encounter rate constant $\kappa$ in a simple encounter process of the type $A+B \rightarrow A B$ was first performed in 1916 by Smoluchowski (1916, 1917) under the hypothesis of chemically isotropic reactants. ${ }^{1}$ In a first approximation, he treated the two reacting species $\mathrm{A}$ and $\mathrm{B}$ as spheres of radius $R_{1}$ and $R_{2}$ with reactivity homogeneously distributed over their surface. In this case one obtains the Smoluchowski encounter rate constant

$\kappa_{\mathrm{s}}=4 \pi D R$,

where $D=D_{1}+D_{2}$ is the coefficient of relative diffusion and $R=R_{1}+R_{2}$ is the encounter distance.

In reactions of the type in Eq. 1, it is often assumed that the second stage proceeds faster with respect to the characteristic time scales of separation of the unreacted encounter pair. In this case, the overall process will be rate-limited by diffusion, and the overall rate of the reaction will equal the encounter rate. For example, this conclusion may be expected to hold truer and truer for antigens of larger and larger size. If that is not the case, one also needs both the rate of dissociation of the unreacted complex and the rate of fixation of the antigenantibody complex in order to make a comparison with the experimental rates. However, it is at present still unclear whether the formation of the stable antigenantibody complex is always a diffusion-controlled reaction (Tanimoto and Kitano 1993). As a matter of fact, experimental measurements of the rate constants in solution for immunological systems including proteins as antigens gave values $10^{-4}$ times smaller than expected from the theory of diffusion (Kitano et al. 1987; Levison et al. 1968; Noble et al. 1969), thus suggesting that the process should be better modeled as an activation-controlled reaction. Furthermore, measurements at the liquid-solid interface show conflicting results, with values only 10 times smaller than the theoretical estimates (Tanimoto and Kitano 1993) as well as of the same order of the values measured in solution (Loset et al. 2004). Importantly, however, all these estimates have been

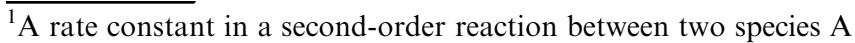
and $\mathrm{B}$ that is first order in $\rho_{\mathrm{A}}$ and first order in $\rho_{\mathrm{B}}$ is usually expressed in moles per decimeter cubed per second $\left(\mathrm{M}^{-1} \mathrm{~s}^{-1}\right)$.
}

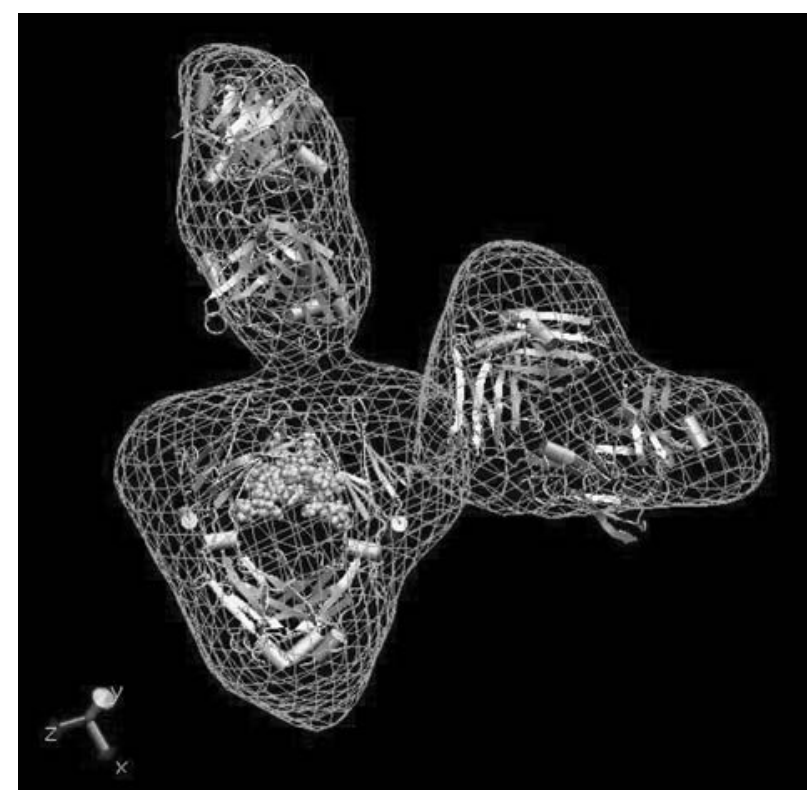

Fig. 1 Pseudoatomic model of a murine immunoglobulin $G$ reconstructed by cryo-electron tomography. The (modified) crystal structure is shown docked into the tomograms (wire frames). Copyright (2004) National Academy of Sciences, USA

compared with the simple Smoluchowski theory (Eq. 2), by treating antibodies as uniformly absorbing spheres of given radius. In general, however, chemical reactions usually display strongly anisotropic behavior, and antibodies are no exception. It appears therefore important to quantify the reduction in the encounter rate due to steric effects within the framework of a simple but realistic model.

Mathematically, reactions between anisotropic agents are described by mixed boundary value problems, for which exact solutions can be found only in some simple cases. A number of approximate solutions extending the simple theory of Smoluchowski were later worked out by many authors to study a variety of problems of diffusion-controlled reactions between a chemically isotropic sphere and a chemically anisotropic one, whose surface is only partially allowed to react (Barzykin and Shushin 2001). Theoretical calculations have also been compared to and integrated with a variety of simulations, ranging from standard methods such as Brownian dynamics (Potter et al. 1996) to more refined tools, such as numerical algorithms to study reactions in biomolecular systems using the equations of continuum mechanics (Song et al. 2004).

Quite unintuitively, it has been established that the encounter rate scales as the linear dimension of the absorbing region. An exact solution of the mixed boundary value problem for an axially symmetric circular absorbing patch has been given by Traytak (1995), by using the formalism of dual series relations (DSR). Surprisingly enough, by comparing the approximate solutions to the same problem available in the literature, he found that very good agreement with the exact solution was displayed by the simple formula 
$\kappa_{\Delta S}=\kappa_{S} \sqrt{\Delta S / S}$, where $\Delta S / S$ is the absorbing fraction of the surface of one sphere.

In this paper, we follow the DSR approach to calculate the stationary encounter rate constant between a chemically isotropic sphere (the antigen) and a pair of anisotropic spheres with given absorbing surface fraction (the Fabs), placed at a given distance. This model can be regarded as the simplest approximation of the encounter process between an antibody and a foreign agent. Yet, it allows us to study quantitatively the reduction of the encounter rate arising from steric effects, and to compare it with the reduction found in the experiments. Moreover, our model will help elucidate an important feature common to many diffusion-driven reactions in chemistry and biology, namely, the effect of anticooperativity. Given a spherical antigen, two absorbing Fabs will always display an encounter rate greater than that of a single absorbing Fab with the same radius, but smaller than twice as much.

Quite generally, we can imagine that the reduction in the encounter effectiveness caused by the presence of two reacting sites is counterbalanced by advantages of different nature specific to the same arrangement. In other words, we think of the action of nature during evolution as promoting trade-offs between competing selective pressures. In the present case, for example, the reduction of the antigen-antibody encounter rate might be recovered as an increase in the binding strength from two-Fab binding to large antigens (such as proteins on the surface of virus capsids) or from polymerization of antigenantibody complexes (Voet and Voet 1995). It is therefore important to characterize the effect of anticooperativity as a function of the parameters which describe the geometry of the system.

\section{Modeling the antigen-antibody encounter}

Before embarking on the formulation and solution of our mathematical model, let us briefly sketch the outline of our work and anticipate the main results as a guide to the reader through the paper.

Our aim is to model the two-site absorbing system which constitutes the chasing apparatus of an antibody (see the pictorial representation in Fig. 2). There are two key parameters: the active surface fraction on the tip of each Fab and the Fab-Fab distance $d$. If we approximate the arm of each Fab as a sphere, the size of the active site may be expressed in terms of an angular aperture measured from its center. While the latter is a geometrical parameter, the Fab-Fab distance is a dynamical quantity, given the high flexibility of the antibody. As a consequence, the average reactivity of the system will be the result of the statistical superposition of all configurations, each weighted according to its thermodynamical weight. However, for small, highly mobile antigens (roughly up to the size of a Fab), the

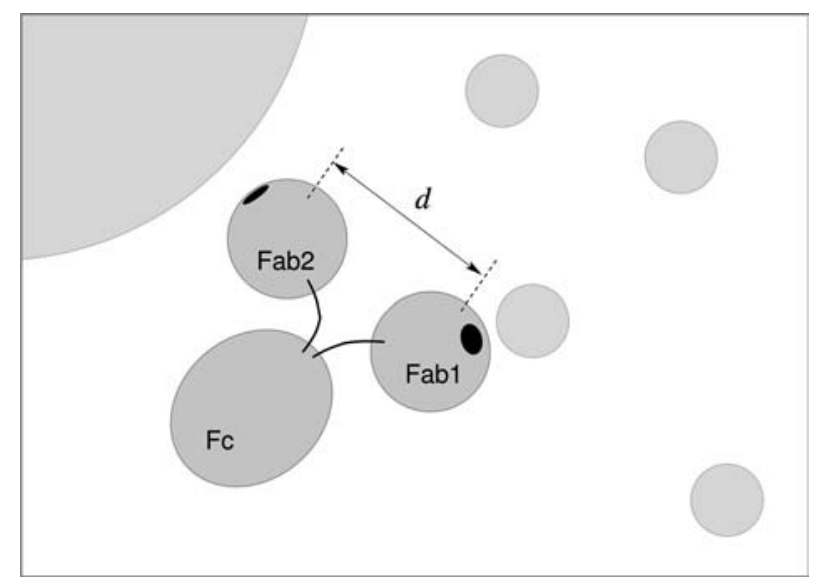

Fig. 2 Schematic view of an antibody facing encounter with antigens of different size. The relative dimensions of the objects roughly reproduce the realistic proportions. The smallest spheres represent small target proteins as the ones relevant for the present analysis. The dark patches on the outer surfaces of the Fabs represent the binding active sites

system may be characterized by the average Fab-Fab distance and all reaction indicators may be regarded in this regime as static ones. For the sake of simplicity, the forthcoming discussion is carried out under these conditions.

The main concern of this paper is to provide a quantitative characterization of the encounter efficiency of a simplified two-Fab system. For this purpose, we formulate an essential mathematical model, which is described in the section "The mathematical formulation". We look for solutions in the case of spherical Fabs with axially symmetric active patches pointing toward opposite directions (see also Fig. 3). This is a reasonable guess that captures the nature of the average patchpatch relative orientation. Clearly, a realistic antibody will adopt conformations that deviate from this guess. However, the main results of our analysis will not be substantially modified for small deviations from the axially symmetric geometry.

In the section "Encounter rate for two entirely absorbing Fabs", we solve the model under the hypothesis of large active sites. We show that the two objects systematically act in an anticooperative fashion, thereby proving that the two-Fab geometry of antibodies sacrifices encounter effectiveness to the possible alternative strategy of twice as many single-Fab agents. If $\kappa$ and $\kappa_{1}$ indicate the rate of the composite and singlecenter systems, respectively, we show that for small antigens of radius $R$ one has

$\frac{\kappa}{2 \kappa_{1}} \approx 1-\left(\frac{R_{\mathrm{F}}+R}{d}\right)$,

where $R_{\mathrm{F}}$ is the Fab radius. The message is that although some encounter effectiveness is lost with twoFab antibodies with respect to twice as many imaginary single-center ones $\left(\kappa<2 \kappa_{1}\right)$, some of it may be regained by letting the molecule keep the active centers as far 
apart as possible (Fig. 2). This conclusion fits nicely within the reconstruction of the $\mathrm{IgG}$ dynamics reported recently by Bongini et al. (2004), where an antibody was shown to display a statistical preference for open configurations. A simple indicator that measures anticooperativity is introduced at this level of the discussion.

We then move in the sections "Encounter rate for two partially absorbing Fabs" and "Anticooperativity of two partially absorbing Fabs" to study the effects of reducing the size of the binding sites. By solving our simplified model, we shall provide quantitative evidence that the anticooperative effect is reduced in molecules with two highly anisotropic binding domains. This conclusion can be grasped intuitively by thinking of anticooperativity as arising because each Fab shields some antigen flux away from its neighbor. This result suggests that the reduction of the anticooperative effect brought about by the unavoidable high binding anisotropy may have positively biased nature toward two-Fab antibodies. From a mathematical point of view, we are able to cast this effect in simple terms by an effective description that is embodied in Eq. 51.

We stress that the final result of our calculations enables us to estimate the absolute value of the antigenantibody encounter rate, provided a sensible estimate of the binding areas is known. However, the latter is difficult to evaluate - indeed it probably constitutes matter for a dedicated study on its own right.

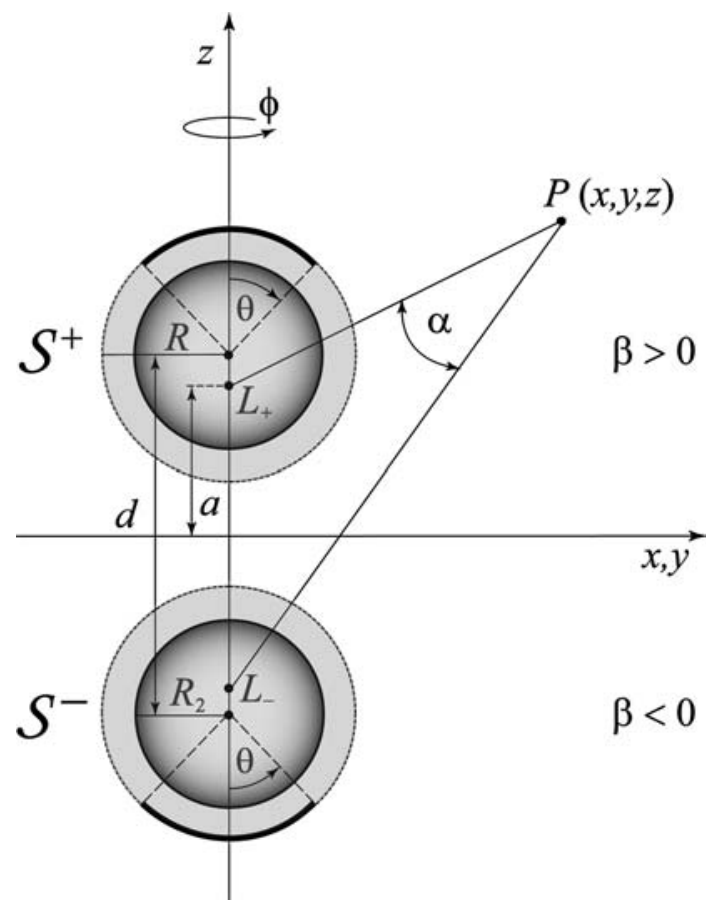

Fig. 3 The system of bispherical coordinates $\beta, \alpha, \phi$. Sections of the two reacting spheres $\mathcal{S}^{ \pm}$are shown with their absorbing patches

\section{The mathematical formulation}

We are interested in studying the encounter rate of a pair of identical Fab arms with an antigen of given size. In the simplest approximation, we neglect the contribution of the nonreacting $\mathrm{Fc}$ stem and assume isotropic antigens. We thus model the immunoglobulin as a pair of partially reacting spheres and the antigen as a uniformly reacting sphere. Let $\mathcal{S}_{1}$ represent the isotropic antigen radius $R_{1}$ and $\mathcal{S}_{2}^{+}, \mathcal{S}_{2}^{-}$indicate the pair of Fab spheres of radius $R_{2}$. Let $\mathbf{R}^{ \pm}$indicate the position vectors of the Fab spheres and $\mathbf{R}_{\mathrm{a}}$ the position vector of the antigen. The diffusion equation then takes the following form:

$$
\begin{aligned}
\frac{\partial P\left(\mathbf{R}^{ \pm}, \mathbf{R}_{\mathrm{a}}, t\right)}{\partial t}= & D_{1} \nabla_{R_{\mathrm{a}}}^{2} P\left(\mathbf{R}^{ \pm}, \mathbf{R}_{\mathrm{a}}, t\right) \\
& +D_{2}\left[\nabla_{\mathbf{R}^{+}}^{2}+\nabla_{\mathbf{R}^{-}}^{2}\right] P\left(\mathbf{R}^{ \pm}, \mathbf{R}_{\mathrm{a}}, t\right)
\end{aligned}
$$

where $D_{1}$ is the diffusion coefficient of the antigen and $D_{2}$ that of a Fab sphere.

In the following we shall neglect time-dependent effects. We thus restrict ourselves to finding the stationary solution of Eq. 3 with the proper choice of boundary conditions. This means that our result will describe with greater accuracy the limit of antigens small with respect to the Fab arms. Let us perform the following coordinate transformation:

$$
\left\{\begin{array}{l}
\mathbf{R}_{\mathrm{G}}=\sqrt{\frac{2 D_{1}}{D_{2}}}\left(\frac{\mathbf{R}^{+}+\mathbf{R}^{-}}{2}\right)+\sqrt{\frac{2 D_{2}}{D_{1}}} \mathbf{R}_{\mathrm{a}} \\
\mathbf{d}=\mathbf{R}^{+}-\mathbf{R}^{-} \\
\mathbf{r}=\mathbf{R}_{\mathrm{a}}-\left(\frac{\mathbf{R}^{+}+\mathbf{R}^{-}}{2}\right) .
\end{array}\right.
$$

The vector $\mathbf{R}_{\mathrm{G}}$ describes the "diffusional" center of mass of the system composed by the pair of Fabs and the antigen, $\mathbf{d}$ is the relative position of one Fab with respect to the other, and $\mathbf{r}$ is the relative position of the antigen with respect to the center of mass of the two-Fab system (clearly a distance $|\mathbf{d}| / 2$ away from each Fab along d). The constraint $d=|\mathbf{d}|=$ const. is assumed. As a result of the transformation (Eq. 4), the stationary diffusion equation becomes

$$
\begin{aligned}
D^{*} \nabla_{\mathbf{R}_{\mathrm{G}}}^{2} P\left(\mathbf{R}_{\mathrm{G}}, \mathbf{r}, \mathbf{d}\right) & +D * \nabla_{\mathrm{r}}^{2} P\left(\mathbf{R}_{\mathrm{G}}, \mathbf{r}, \mathbf{d}\right) \\
& +2 D \nabla_{\mathrm{d}}^{2} P\left(\mathbf{R}_{\mathrm{G}}, \mathbf{r}, \mathbf{d}\right)=0,
\end{aligned}
$$

where $D^{*}=\left(D_{1}+D_{2}\right) / 2$. We observe that the last operator in Eq. 5 is a purely angular one, as a result of the constraint $d=$ const.

Equation 5 can be considered as describing three independent diffusion processes, one in the coordinate $\mathbf{R}_{\mathrm{G}}($ translational diffusion of the whole system), one in the coordinate $\mathbf{r}$ (translational diffusion of the antigen with respect to the antibody), and one in the coordinate d (rotational diffusion of the two Fab spheres on the sphere centered in their barycenter). Thus the distribution function $P$ may be factorized as 
$P\left(\mathbf{R}_{\mathrm{G}}, \mathbf{r}, \mathbf{d}\right)=\mathcal{P}\left(\mathbf{R}_{\mathrm{G}}\right) c(\mathbf{r}) p(\mathbf{d})$. If one disregards the diffusion along the coordinate $\mathbf{R}_{\mathrm{G}}$, the relevant remaining relative motion is governed by the following equation:

$D^{*}\left[\frac{\nabla_{\mathrm{r}}^{2} c(\mathbf{r})}{c(\mathbf{r})}\right]+2 D_{2}\left[\frac{\nabla_{\mathrm{d}}^{2} p(\mathbf{d})}{p(\mathbf{d})}\right]=0$.

The two terms in Eq. 6 must be constant. In particular they must be equal to the same quantity. However, there are no boundary conditions to be imposed on the rotational diffusion of the two Fabs on the spherical surface centered at their center of mass with radius $d / 2$. This means that the function must have ssymmetry and hence $p(\mathbf{d})=1 / 4 \pi$. We are then left with a Laplace problem of the form $\nabla^{2} c(\mathbf{r})=0$ with the proper set of mixed boundary conditions which describe the anisotropy of the antigen-antibody encounter.

The reaction between an antigen and a Fab takes place on the given active surface fraction of either sphere $\mathcal{S}^{ \pm}$of radius $R$, concentric with $\mathcal{S}_{2}^{ \pm}$(Fig. 3). The rest of the surface of both Fabs is taken to be reflecting. Under these hypotheses, our task is to solve the following mixed boundary value Laplace problem for the concentration of antigens $c(\mathbf{r})$ :

$\nabla^{2} c(\mathbf{r})=0$,

$\left.c\right|_{\Omega_{+}}=\left.c\right|_{\Omega_{-}}=0$,

$\left.\vec{\nabla} c \cdot \hat{n}_{+}\right|_{\hat{\Omega}_{+}}=\left.\vec{\nabla} c \cdot \hat{n}_{-}\right|_{\hat{\Omega}_{-}}=0$,

$\lim _{r \rightarrow \infty} c(\mathbf{r})=c_{\infty}$,

where $\Omega_{ \pm}$denote the absorbing fractions of the spheres $\mathcal{S}^{ \pm}, \quad \hat{n}_{ \pm}$the outward normals to their surfaces, $\Omega_{ \pm}$the complementary reflecting surface fractions and $c_{\infty}$ the bulk concentration of the antigens.

The Laplace equation is known to be separable in many coordinate systems (Morse and Feshbach1953). The nature of the problem at hand suggests the use of the bispherical coordinate set, defined by the three variables $(\beta, \alpha, \phi)$ (Fig. 3):

$$
\begin{aligned}
& \beta=-\log \left(\frac{P L_{+}}{P L_{-}}\right) \quad \text { with }-\infty<\beta<\infty \\
& \alpha=\left(\widehat{L}_{-} \widehat{P L}_{+}\right) \quad \text { with } 0 \leqslant \alpha \leqslant \pi .
\end{aligned}
$$

The azimuthal angle $\phi$ is chosen such that $\phi=0$ if $P$ belongs to the $x$-axis $(0 \leq \phi \leq 2 \pi)$. Bispherical coordinates are related to Cartesian coordinates through the following transformations:

$$
\begin{aligned}
& x=\frac{a \sin \alpha \cos \phi}{\cosh \beta-\cos \alpha} \\
& y=\frac{a \sin \alpha \sin \phi}{\cosh \beta-\cos \alpha} \\
& y=\frac{a \sinh \beta}{\cosh \beta-\cos \alpha} .
\end{aligned}
$$

The parameter $a$ is fixed by the distance $d$ between the centers of the absorbing spheres and by their radius $R$. In our case, we have
$a=R \sqrt{\chi^{2}-1}$,

with $\chi=d / 2 R>1$. The two poles $(\beta \rightarrow \pm \infty)$ are located on the $z$-axis at the points $L_{+}$and $L_{-}$(Fig. 3), at a distance $a$ from the plane $z=0$. The surfaces $\beta=\beta_{0}=$ const. are spheres of radius $a \operatorname{cosech}\left|\beta_{0}\right|$, whose centers are located on the $z$-axis at $z= \pm \operatorname{coth} \beta_{0}$. The surface $\beta=0$ is a sphere of infinite radius with center at $(x=y=0, z= \pm \infty)$, which includes the plane $z=0$. In particular, the two reaction spheres $\mathcal{S}^{ \pm}$are defined as $\beta= \pm \beta_{0}$, with

$\beta_{0}=\log \left(\chi+\sqrt{\chi^{2}-1}\right)$.

From Eq. 14the following two identities can be straightforwardly derived:

$\cosh \beta_{0}=\chi, \quad \sinh \beta_{0}=\sqrt{\chi^{2}-1}$.

The absorbing surface fractions of the spheres $\mathcal{S}^{ \pm}$are defined as circular patches of given angular aperture $\theta=\theta_{0}, \theta$ being the latitude measured from the north $\left(\mathcal{S}^{+}\right)$and south $\left(\mathcal{S}^{-}\right)$pole of the reacting spheres (Fig. 3),

$\Omega_{+}=\left(\beta=\beta_{0}, 0 \leqslant \alpha \leqslant \alpha_{0}, 0 \leqslant \phi \leqslant 2 \pi\right)$,
$\Omega_{-}=\left(\beta=-\beta_{0}, 0 \leqslant \alpha \leqslant \alpha_{0}, 0 \leqslant \phi \leqslant 2 \pi\right)$,

where

$\alpha_{0}=\arccos \left[\frac{\chi \sin ^{2} \theta_{0}+\left(\chi^{2}-1\right) \cos \theta_{0}}{\chi^{2}-\cos ^{2} \theta_{0}}\right]$.

We note that the parameters $a$ and $\beta_{0}$ completely describe the geometry of the problem. All of the space is divided into three parts: that within sphere $\mathcal{S}^{+}\left(\beta>\beta_{0}\right)$, that within $\mathcal{S}^{-}\left(\beta<-\beta_{0}\right)$, and that outside of both spheres $\left(-\beta_{0}<\beta<\beta_{0}\right)$.

As a final remark, we observe that the limits $r \rightarrow 0$ and $r \rightarrow \infty$ are obtained in bispherical coordinates by letting $(\beta \rightarrow 0, \alpha \rightarrow \pi)$ and $(\beta \rightarrow 0, \alpha \rightarrow 0)$, respectively.

\section{Laplace equation in bispherical coordinates}

The most general solution to the Laplace equation in bispherical coordinates in a problem with azimuthal symmetry satisfying Eq. 10 can be written as (Morse and Feshbach1953)

$$
\begin{aligned}
\rho(\beta, \alpha)= & 1-\sqrt{\cosh \beta-\cos \alpha} \\
& \times \sum_{n=0}^{\infty}\left(A_{n} \mathrm{e}^{[n+(1 / 2)] \beta}+B_{n} \mathrm{e}^{[n+(1 / 2)] \beta}\right) P_{n}(\cos \alpha),
\end{aligned}
$$

where $P_{n}(\cos \alpha)$ are the Legendre polynomials, and we have introduced the normalized concentration $\rho=c / c_{\infty}$.

Once the constants $A_{n}$ and $B_{n}$ have been determined by imposing Eqs. 8 and 9 the calculation of the encounter rate constant can be performed by evaluating 
the flux $\Phi$ of the concentration (Eq. 18) on any surface enclosing the two sinks $\Omega_{ \pm}$. Since we are assuming homogeneous bulk concentration of the antigens, the rate calculated at any orientation of the vector $\mathbf{d}$ must give the same result. Therefore, a sensible choice for the calculation of the rate is to take the flux across the very spheres $\beta= \pm \beta_{0}$. Letting $\kappa$ be the rate constant of encounters between a spherical antigen and the twosphere system with given absorbing fraction, we can write

$$
\begin{aligned}
\kappa & =D^{*} \Phi(\rho) \\
& =D^{*}\left(\int_{\Omega_{+} \cup \hat{\Omega}_{+}} \vec{\nabla} \rho \cdot \hat{n}_{+} \mathrm{d} S+\int_{\Omega_{-} \cup \hat{\Omega}_{-}} \vec{\nabla} \rho \cdot \hat{n}_{-} \mathrm{d} S\right) .
\end{aligned}
$$

This choice of the integration surface allows the expression of the flux to be cast in a simple form, by noting that $\hat{n}_{ \pm}=\mp \hat{u}_{\beta}$, where $\hat{u}_{\beta}$ is the unit vector perpendicular to the surfaces $\beta=$ const. We have

$$
\begin{aligned}
\kappa=D^{*} & {\left[-\int_{\Omega_{+} \cup \hat{\Omega}_{+}}\left[\nabla \rho\left(\beta_{0}, \alpha\right)\right]_{\beta} \mathrm{d} S\right.} \\
& \left.+\int_{\Omega_{-} \cup \hat{\Omega}_{-}}\left[\nabla \rho\left(-\beta_{0}, \alpha\right)\right]_{\beta} \mathrm{d} S\right],
\end{aligned}
$$

where

$$
\left[\nabla \rho\left( \pm \beta_{0}, \alpha\right)\right]_{\beta}=\left.\left(\frac{\cosh \beta_{0}-\cos \alpha}{a}\right) \frac{\partial \rho}{\partial \beta}\right|_{ \pm \beta_{0}}
$$

is the $\beta$ component of the gradient of the concentration $\rho$ evaluated on the spherical surfaces $\beta= \pm \beta_{0}$.

The infinitesimal surface element d $S$ can be calculated in a simple fashion by parameterizing the surfaces $\beta= \pm \beta_{0}$ as $\psi(u, v): \mathbb{U} \rightarrow \mathbb{R}_{\nvdash}, \mathbb{U} \subset \mathbb{R}_{\nvdash}$ with the choice $(u, v)=(\alpha, \phi) \mathbb{U}=[\nvdash, \pi] \cup[\nvdash, \nvdash \models \pi]$.In this case, we have

$\mathrm{d} S=\left|\partial_{u} \psi \wedge \partial_{v} \psi\right| \mathrm{d} u \mathrm{~d} v=\frac{a^{2} \sin u}{\left(\cosh \beta_{0}-\cos u\right)^{2}} \mathrm{~d} u \mathrm{~d} v$.

By inserting Eqs. 21 and 22 into Eq. 20, the integration over $\phi$ can be performed straightforwardly. We get

$$
\kappa=-2 \pi D^{*} a \int_{0}^{\pi}\left(\left.\frac{\partial \rho}{\partial \beta}\right|_{\beta_{0}}-\left.\frac{\partial \rho}{\partial \beta}\right|_{-\beta_{0}}\right) \frac{\sin \alpha}{\cosh \beta_{0}-\cos \alpha} \mathrm{d} \alpha .
$$

\section{Encounter rate for two entirely absorbing Fabs}

In this section we shall work out the encounter rate for the case of two entirely absorbing Fabs $\left(\theta_{0}=\alpha_{0}=\pi\right)$. We have a single boundary value problem to solve now, namely,

$$
\begin{aligned}
& \nabla^{2} c(\mathbf{r})=0, \\
& \left.c\right|_{\mathcal{S}^{+}}=\left.c\right|_{\mathcal{S}^{-}}=0,
\end{aligned}
$$

$\lim _{r \rightarrow \infty} c(\mathbf{r})=c_{\infty}$.

In order to develop the calculations, it is useful to introduce the following identity (Morse and Feshbach1953):

$\frac{1}{\sqrt{\cosh \beta-\cos \alpha}}=\sqrt{2} \sum_{n=0}^{\infty} \mathrm{e}^{[n+(1 / 2)]|\beta|} P_{n}(\cos \alpha)$,

where $P_{n}(\cos \alpha)$ are Legendre polynomials. The unknown coefficients $A_{n}$ and $B_{n}$ in the general expression (Eq. 18) can be determined by imposing Eq. 25. If we take into account Eq. 27 and invoke the uniqueness of development in series of Legendre polynomials, we get the following algebraic system,

$$
\left\{\begin{array}{l}
A_{n} \mathrm{e}^{(2 n+1) \beta_{0}}+B_{n}=\sqrt{2} \\
A_{n}+B_{n} \mathrm{e}^{(2 n+1) \beta_{0}}=\sqrt{2}
\end{array},\right.
$$

whose solutions are easily found to be

$A_{n}=B_{n}=\frac{1}{\sqrt{2}} \frac{\mathrm{e}^{-[n+(1 / 2)] \beta_{0}}}{\cosh \left[\left(n+\frac{1}{2}\right) \beta_{0}\right]}$.

If we substitute back this expression for the coefficients in Eq. 18, we obtain the normalized solution of the Laplace problem (Eqs. 24, 25, 26):

$$
\begin{aligned}
\rho(\beta, \alpha)= & 1-\sqrt{\cosh \beta-\cos \alpha} \\
& \times \sum_{n=0}^{\infty} \frac{\cosh \left[\left(n+\frac{1}{2}\right) \beta\right]}{\cosh \left[\left(n+\frac{1}{2}\right) \beta\right]} \mathrm{e}^{-\left(n+\frac{1}{2}\right) \beta_{0}} P_{n}(\cos \alpha) .
\end{aligned}
$$

The encounter rate constant can now be calculated using Eq. 23. The details of the calculations are reported in "Appendix 1". We obtain

$\kappa=2 \kappa_{\mathrm{S}} \sqrt{\chi^{2-1}} \sum_{n=0}^{\infty} \frac{2}{1+\left(\chi+\sqrt{\chi^{2}-1}\right)^{2 n+1}}$,

where $\chi=d / 2 R$ and $\kappa_{\mathrm{S}}=4 \pi D^{*} R$ is the Smoluchowski rate constant describing the encounter of an antigen and a single Fab with diffusion coefficient $D_{2} / 2$.

For large values of $\chi$, a careful calculation allows us to develop Eq. 31. To first order in $1 / \chi$, we obtain

$\kappa=2 \kappa_{\mathrm{S}}\left(1-\frac{1}{2 \chi}\right)=2 \kappa_{\mathrm{S}}\left(1-\frac{R}{d}\right)$.

In the limit of infinite separation between the two Fabs, we have

$\lim _{\chi \rightarrow \infty} \kappa=2 \kappa_{\mathrm{S}}$

and we recover additivity of two noninteracting absorbing spheres. We observe that, as a result of our formulation, in the limit $d \rightarrow \infty$ the relative motion of the antigen and the Fabs is still described by the sum of the diffusion coefficient of the antigen $D_{1}$ and that of the two-Fab system $D_{2} / 2$. 
In the opposite limit $\chi \rightarrow 1$, i.e. when the two reacting spheres $\mathcal{S}^{ \pm}$are brought into contact, the rate (Eq. 31) can be evaluated analytically by letting $\chi=\cosh \delta$. We have

$$
\begin{aligned}
\lim _{\chi \rightarrow 1} \frac{\kappa}{2 \kappa_{\mathrm{S}}} & =\lim _{\delta \rightarrow 0} \sum_{n=0}^{\infty} \frac{2 \delta \Delta n}{1+\mathrm{e}^{(2 n+1) \delta}} \\
& =\int_{0}^{\infty} \frac{\mathrm{d} x}{1+\mathrm{e}^{x}}=\log 2 .
\end{aligned}
$$

Numerical calculation of $\kappa$ through Eq. 31 shows that the approximation (Eq. 32) is accurate within about $2 \%$ for values of $\chi$ as small as $\chi \approx 4$.

Equation 31 describes the encounter between a spherical antigen and two entirely absorbing Fabs a distance $d$ apart. The rate constant $\kappa$ of such a reaction is always smaller than $2 \kappa_{\mathrm{S}}$, which amounts to saying that the two Fabs behave in an anticooperative fashion. The physical reason for such behavior is that, for any finite value of $d$, the two Fab spheres partially screen each other from a finite portion of the flux of incoming antigens. A quantitative measure of the anticooperativity can be obtained by defining the simple function

$\mathcal{A}=\frac{\kappa}{2 \kappa_{1}}-1$,

where $\kappa_{1}=4 \pi\left(D_{1}+D_{2}\right)\left(R_{1}+R_{2}\right)$ is the Smoluchowski rate constant describing the encounter of the antigen with a single, isolated Fab sphere. If we use the Einstein rule $D_{i} \propto 1 / R_{i}, i=1,2$, the anticooperativity function can be written as

$\mathcal{A}=\frac{1}{2}\left(\frac{2+\xi}{1+\xi}\right) \frac{\kappa}{2 \kappa_{\mathrm{S}}}-1$

where $\xi=R_{1} / R_{2}$ is the antigen-to-antibody size ratio.

The quantity $\mathcal{A}$ is a monotonously decreasing function of the antigen radius $R_{1}$ for a fixed Fab-Fab separation $d$, and is a monotonously increasing function of $d$ at fixed $R_{1}$ (Fig. 4). This means that the configurations of the IgG with the largest Fab-Fab distances will be

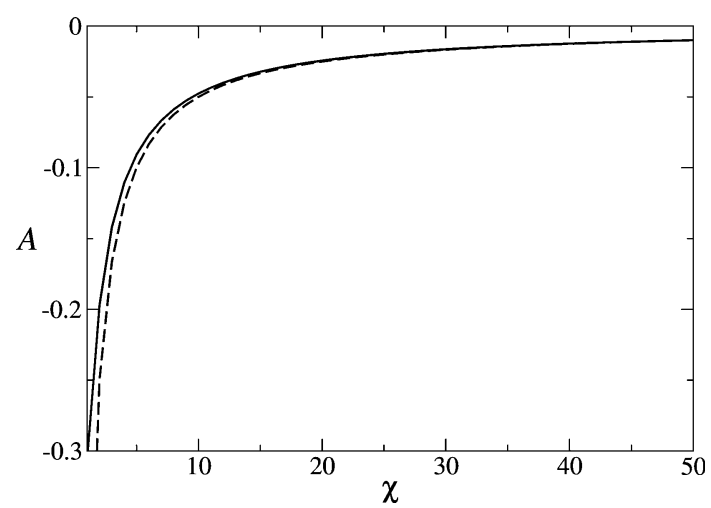

Fig. 4 Anticooperativity indicator $\mathcal{A}$ as a function of $\chi$ for a system of two entirely absorbing spherical Fabs. The solid line is a numerical calculation using Eq. 31. The dashed line is a plot of the first-order approximation using Eq. 32 characterized by the smallest values of anticooperativity. In the formal limit of infinite separation between active centers $d \rightarrow \infty$, additivity is nearly recovered. For small antigens

$\lim _{d \rightarrow \infty} \mathcal{A}(\chi)=-\frac{\xi}{1+\xi} \ll 1$.

Of course, additivity is exactly recovered only formally in the limit $\xi \rightarrow 0$.

In the opposite limit $\chi \rightarrow 1$, anticooperativity attains its maximum. In this case $\lim _{\chi \rightarrow 1} \kappa=2 \kappa_{\mathrm{s}} \log 2$, and hence

$\lim _{\chi \rightarrow \infty} \mathcal{A}(\chi) \frac{1}{2}\left(\frac{2+\xi}{1+\xi}\right) \log 2-1$

We see that for antigens as small as $\xi=0.1$, $\lim _{\gamma \rightarrow 1} \mathcal{A} \approx 0.95 \log 2-1 \approx-0.34$. This means that two entirely absorbing Fabs may lose as much as 35\% of their combined encounter effectiveness when they happen to be very close to each other.

\section{Encounter rate for two partially absorbing Fabs}

We turn now to a more realistic case, where we allow for only a given fraction of the surface of either Fab to be active, according to Eqs. 16 and 17. The rest of the surface is taken as reflecting. As a consequence, we are faced with the full mixed boundary value problem (Eqs. 7, 8, 9, 10).

Substitution of Eq. 18 in Eqs. 8 and 9, with the help of the identity (Eq. 27), leads to the following DSR

$\sum_{n=0}^{\infty}\left\{A_{n} \cosh \left[\left(n+\frac{1}{2}\right) \beta_{0}\right]-q_{n}\right\} P_{n}(\cos \alpha)=0$

for $0<\alpha<\alpha_{0}$,

$\sum_{n=0}^{\infty}(2 n+1) A_{n} \sinh \left[\left(n+\frac{1}{2}\right) \beta_{0}\right] P_{n}(\cos \alpha)+\frac{\sinh \beta_{0}}{\cosh \beta_{0}-\cos \alpha}$

$\times \sum_{n=0}^{\infty} A_{n} \cosh \left[\left(n+\frac{1}{2}\right) \beta_{0}\right] P_{n}(\cos \alpha)=0$

for $\alpha_{0}<\alpha<\pi$,

where $\alpha_{0}$ is given by Eq. $17, B_{n}=A_{n}$ and

$q_{n}=\frac{1}{\sqrt{2}} \mathrm{e}^{-\left(n+\frac{1}{2}\right) \beta_{0}}$.

The DSR (Eqs. 37, 38) can be reduced to a form solvable by iteration through the definition of new unknown coefficients $X_{n}$ :

$X_{n}=A_{n} \cosh \left[\left(n+\frac{1}{2}\right) \beta_{0}\right]-q_{n}$.

After some elementary algebra, the DSR (Eqs. 37, 38) can be rearranged in the following fashion: 


$$
\left\{\begin{array}{cc}
\sum_{n=0}^{\infty} X_{n} P_{n}(\cos \alpha)=0 & \text { for } 0 \leqslant \alpha \leqslant \alpha_{0} \\
\sum_{n=0}^{\infty}(2 n+1) X_{n} P_{n}(\cos \alpha)=G(\alpha) & \text { for } \alpha_{0} \leqslant \alpha \leqslant \pi
\end{array},\right.
$$

where

$$
\begin{aligned}
G(\alpha)= & \sum_{n=0}^{\infty}(2 n+1)\left\{X_{n}-\left(q_{n}+X_{n}\right) \tanh \left[\left(n+\frac{1}{2}\right) \beta_{0}\right]\right\} \\
& P_{n}(\cos \alpha)-\frac{\sinh \beta_{0}}{\cosh \beta_{0}-\cos \alpha} \sum_{n=0}^{\infty}\left(q_{n}+X_{n}\right) P_{n}(\cos \alpha) .
\end{aligned}
$$

It may be shown that a pair of DSR of the form of Eq. 41 have the exact solution (Sneddon 1966)

$X_{n}=\frac{1}{\pi \sqrt{2}} \int_{\alpha_{0}}^{\pi} \cos \left[\left(n+\frac{1}{2}\right) u\right] G_{0}^{*}(u) \mathrm{d} u$

with

$G_{0}^{*}(u)=\int_{u}^{\pi} \frac{\mathrm{G}(\alpha) \sin \alpha}{\sqrt{\cos u-\cos \alpha}} \mathrm{d} u$.

${ }^{2}$ Using Eq. 43 and recalling the definition of $\beta_{0}$ (Eq. 14), we get after a somewhat lengthy calculation the following infinite set of linear equations (see "Appendix 2" for the details):

$X_{n}-\sum_{m=0}^{\infty} M_{n m} X_{m}=\sum_{m=0}^{\infty}\left(M_{n m}-S_{n m}\right) q_{m}$,

where the matrices Sand $\mathbf{M}$ are given by

$$
\begin{aligned}
& S_{n m}=\frac{1}{\pi}\left\{\left(\pi-\alpha_{0}\right) \delta_{n m}-\frac{\sin \left[(n-m) \alpha_{0}\right]}{n-m}\left(1-\delta_{n m}\right) \frac{\sin \left[(n+m+1) \alpha_{0}\right]}{n+m+1}\right\}, \\
& M_{n m}=\frac{2 S_{n m}}{1+\left(\chi+\sqrt{\chi^{2}-1}\right)^{2 m+1}}-\sqrt{\chi^{2}-1} \sum_{k=0}^{\infty} \Gamma_{n k} S_{k m},
\end{aligned}
$$

with

$\Gamma_{m n}=P_{m}(\chi) Q_{n}(\chi) \Theta(n-m)+P_{n}(\chi) Q_{m}(\chi) \Theta(m-n)$,

$Q_{n}$ being Legendre polynomials of the second kind and $\Theta(x)$ the Heaviside step function defined such that $\Theta(0)=1 / 2$.

The set of Eqs. 45 may be solved numerically by truncation. Fortunately, the norm of the matrix $\mathbf{M}$ is less than unity for all values of $\theta_{0}$ and $\chi$ (Fig. 5) and thus the procedure converges rapidly to a fixed point (see "Appendix 3" for more details). Once the coefficients $X_{n}$ have been determined for a given choice of $\theta_{0}$ and $\chi$, the rate may be calculated through Eq. 23. Recalling the definition Eq. 39, we get

\footnotetext{
${ }^{2}$ We note that in the work by Sneddon (1966), Eq. 43 has the wrong sign on the right-hand side.
}

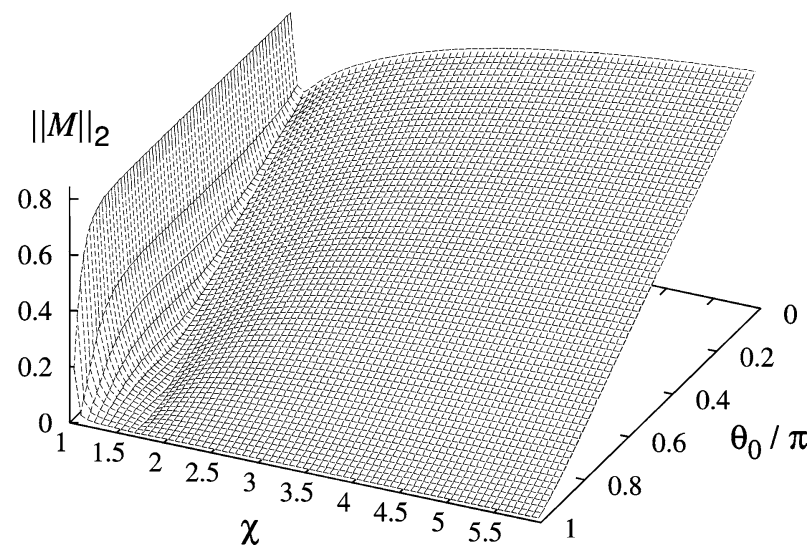

Fig. 5 Surface plot of the spectral norm of matrix $\mathbf{M}$ as a function of the angular aperture of the absorbing patches $\theta_{0}$ and of $\chi=d / 2 R$

$\kappa=2 \kappa_{\mathrm{S}} \sqrt{\chi^{2}-1} \sum_{n=0}^{\infty}\left(1+\frac{\chi_{n}}{q_{n}}\right) \frac{2}{1+\left(\chi+\sqrt{\chi^{2}-1}\right)^{2 n+1}}$.

\section{Anticooperativity of two partially absorbing Fabs}

We have seen that if only a portion of the whole surface of the sinks is allowed to react the rate constant is reduced accordingly. Such reduction in the encounter rate is usually quantified through computation of the socalled steric factor, defined as

$f=\frac{\kappa\left(\theta_{0}\right)}{\kappa(\pi)}$.

A very narrow angular aperture of the two active sites will result in a vanishing $f$, while the value $f=1$ will be approached when the system is everywhere reactive.

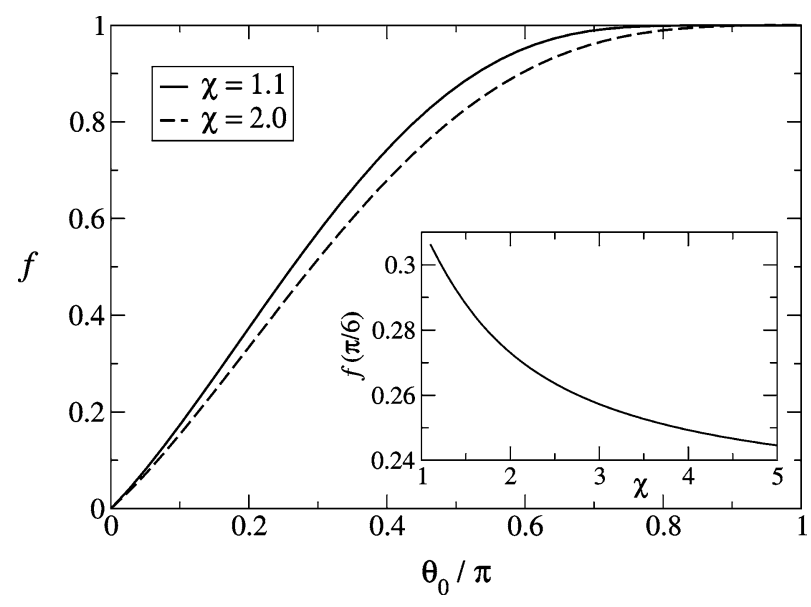

Fig. 6 Steric factor $f$ versus angular aperture of the active regions for two values of $\chi$. In the inset we show the dependence of $f$ upon $\chi$ for $\theta_{0}=\pi / 6$ 
Of course, the steric factor of a pair of patched spheres will depend on the value of the parameter $\chi=d /$ $2 R$. For example, the reduction in the encounter rate constant with antigens of size $R$ caused by restricting the active site to a given aperture $\theta_{0}$ will be different at different values of the Fab-Fab separation $d$. In Fig. 6 we plot the function $f\left(\theta_{0}\right)$ for $\chi=1.1$ and $\chi=2.0$. These values approximately describe the two interesting limiting cases of (1) Fabs close to each other $(\chi=1.1)$ and (2) Fabs placed at maximum separation $(\chi=2.0)$. The latter condition roughly corresponds to the case where $d$ approaches the diameter of the IgG molecule.

Our results clearly show that the encounter rate is greater the smaller is $\chi$ (see in particular the inset in Fig. 6). This fact has a twofold interpretation. One one hand, it means that the anisotropic system, characterized by a given active site, is more and more effective the larger is the antigen. This is the obvious conclusion that holds also in the simple fully reactive arrangement. However, this result also means that the rate of the anisotropic agent increases (with respect to its isotropic
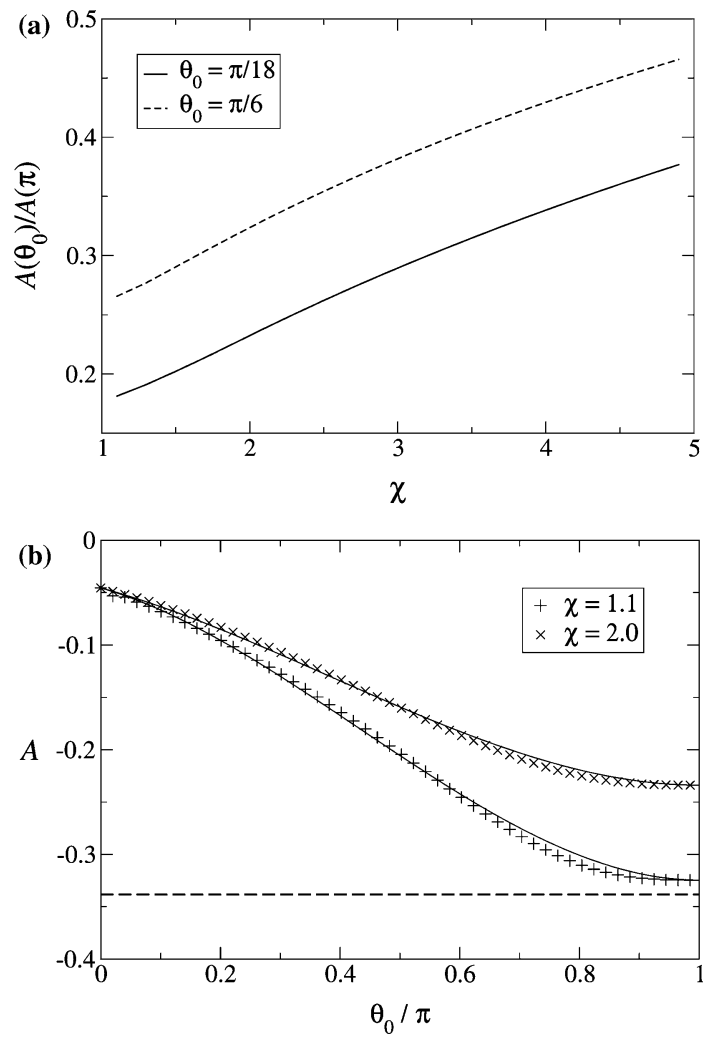

Fig. 7 a Ratio between the anticooperativity of the anisotropic system and that of the fully reactive arrangement versus $\chi$ for two values of the patch aperture $\theta_{0}$. b The anticooperativity function (Eq. 50) versus angular aperture for $\xi=0.1$. The symbolsare the exact solution (Eq. 48), calculated by truncating Eq. 45 at $N=100$. The solid lines are one-parameter fits with the empirical expression (Eq. 51). The best-fit values of the free parameter are $\lambda=-0.164 \pm 0.003(\chi=1), \lambda=-0.156 \pm 0.002(\chi=2)$. The horizontal dashed line marks the maximum of anticooperativity attained by the fully absorbing system in the limit $\chi \rightarrow 1$, namely, $[(2+\xi) / 2(1+\xi)] \log 2-1$ analog) by bringing the two active domains closer (i.e. by decreasing $d$ ).

This effect can be further investigated by introducing the anticooperativity function for the system of two partially reacting Fabs. This will be a function of $\theta_{0}$, namely,

$$
\begin{aligned}
\mathcal{A}\left(\theta_{0}\right) & =\frac{\kappa\left(\theta_{0}\right)}{\kappa(\pi)}-1 \\
& =\frac{1}{2}\left(\frac{2+\xi}{1+\xi}\right) \frac{f\left(\theta_{0}\right)}{f_{1}\left(\theta_{0}\right)}-1,
\end{aligned}
$$

where $\kappa\left(\theta_{0}\right)=2 \kappa_{\mathrm{S}} f\left(\theta_{0}\right)$ is given by Eq. $48, \quad \xi=R_{1} / R_{2}$ and $\quad \kappa_{1}\left(\theta_{0}\right)=4 \pi\left(D_{1}+D_{2}\right)\left(R_{1}+R_{2}\right) f_{1}\left(\theta_{0}\right)$ is the encounter rate for an isolated sphere with an active region of angular size $\theta_{0}$. The latter can be calculated exactly through the DSR approach analogously to what is reported in this paper. Such a solution was first published by Traytak (1995).

It is evident form Fig. 7a that the anisotropic system behaves in a less anticooperative fashion, which is consistent with the results reported in Fig. 6. In particular, this effect is more and more marked for smaller and smaller active sites. In Fig. $7 \mathrm{~b}$ we report the exact calculation of $\mathcal{A}\left(\theta_{0}\right)$ for the two geometries $\chi=1.1$ and $\chi=2.0$ and a reasonable value of the antigen-to-Fab size ratio $\xi=0.1$. The value attained at $\theta_{0}=\pi$ is the anticooperativity of two entirely active Fabs. Our results show that the effect of reducing the reaction region is to reduce the anticooperative behavior. Put differently, when the active site is very small, the negative interference between the two active lobes is maximally reduced. This effect surprisingly increases monotonously with decreasing $\theta_{0}$. Figure 7 summarizes the main result of our analysis.

Unfortunately it is not possible to cast Eq. 50 in a simple analytical form. However, it turns out that a simple third-order polynomial with only one free coefficient fits the exact solution extremely well in the whole domain $[0, \pi]$. The three conditions that fix all the other coefficients are

1. From the numerical calculations it is clear that the steric factors $f\left(\theta_{0}\right)$ and $f_{1}\left(\theta_{0}\right)$ vanish linearly for $\theta_{0} \rightarrow 0$ with the same slope. This fixes the zero coefficient

$$
\mathcal{A}_{0}=\mathcal{A}(0)=\frac{1}{2}\left(\frac{2+\xi}{1+\xi}\right)-1 \text {. }
$$

2. The anticooperativity at $\theta_{0}=\pi$ must be given by Eq. 35, which gives the condition

$$
\begin{aligned}
\mathcal{A}(\pi)= & \mathcal{A}_{\pi} \stackrel{\text { def }}{=} \frac{1}{2}\left(\frac{2+\xi}{1+\xi}\right) \sqrt{\chi^{2}-1} \\
& \times \sum_{n=0}^{\infty} \frac{2}{1+\left(\chi+\sqrt{\chi^{2}-1}\right)^{2 n+1}}-1 .
\end{aligned}
$$

3. The function $\mathcal{A}\left(\theta_{0}\right)$ must approach the value $\mathcal{A}(\pi)$ with slope zero, since both steric factors $f\left(\theta_{0}\right)$ and $f_{1}\left(\theta_{0}\right)$ do so. Again, it is easy to convince oneself of the latter conditions from the numerics. 
If we indicate with $\lambda$ the free first-order coefficient, and work out the three conditions, the empirical oneparameter polynomial anticooperativity takes the form

$$
\begin{aligned}
\mathcal{A}_{\mathrm{e}}\left(\theta_{0}\right)= & \mathcal{A}_{0}+\lambda\left(\frac{\theta_{0}}{\pi}\right)-(2 \lambda-3 \Delta \mathcal{A})\left(\frac{\theta_{0}}{\pi}\right)^{2} \\
& +(\lambda-2 \Delta \mathcal{A})\left(\frac{\theta_{0}}{\pi}\right)^{3}
\end{aligned}
$$

where $\Delta \mathcal{A}_{0}=\mathcal{A}_{\pi}-\mathcal{A}_{0}$.

The result of a fit with Eq. 51 is shown in Fig. 7 for two values of $\chi$. Despite its simple form, it appears to interpolate the exact result extremely well. Accordingly, this approximation may be used at all interesting values of $\chi$. The best-fit values of $\lambda$ turn out to vary linearly with $\chi$ (Fig. 8) as $\lambda \approx-0.176+0.011 \chi$. Finally, this result prescribes which value of $\lambda$ should be used in Eq. 51 if one wishes to compute the anticooperativity at a given value of $\chi$.

\section{Discussion}

In this paper we have derived an explicit formula that allows the steric factor of a molecule composed of two anisotropic binding domains to be calculated as a function of the relevant geometrical parameters. As a case study, we have applied our treatment to the process of encounter between an antibody and a small antigen.

On the basis of our results, we hint that a reduction of a factor $10^{4}$ with respect to the Smoluchowski theory as reported experimentally, if confirmed by more recent work, cannot be justified within the framework of a reaction with an isotropic antigen. The inclusion in our model of anisotropy of the second reactant along the strategy reported by Barzykin and Shushin (2001) will be the subject of future research. However, owing to the discrepancies in the results reported in the literature, we believe that further accurate experimental work is required.

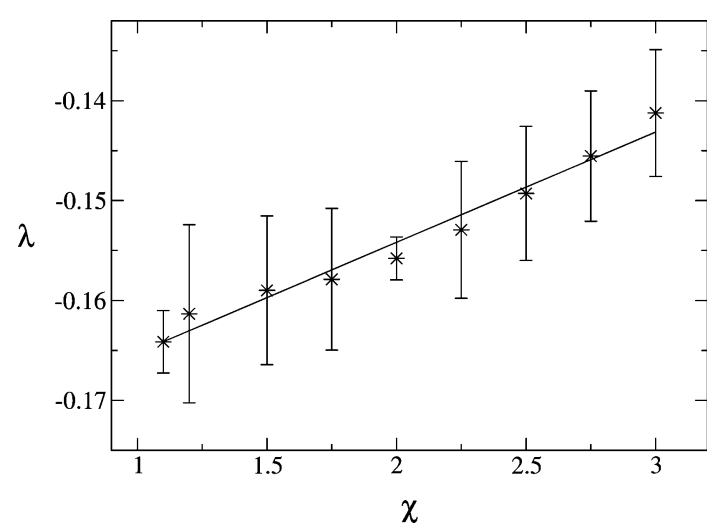

Fig. 8 Best-fit values of $\lambda$ from the fits of the exact anticooperativity with the empirical function (Eq. 51) versus $\chi$ (symbols). The solid line is the linear fit reported in the text
Moreover, our model allows us to show that a further effect naturally arises in a two-sites system, namely, anticooperativity of the two binding centers. We have quantified such an effect by reducing the complex mathematical problem to a simple approximate oneparameter formula that can be employed generically. An important result of our analysis is that the anisotropic system is less anticooperative compared with the fully active arrangement. This effect is more pronounced for small sizes of the active sites.

As to the example of antibodies, it is also interesting to discuss our results from the point of view of evolution. As observed in the "Introduction", we may imagine that nature has eventually come to antibodies with two partially-active domains as a result of a trade-off among contrasting selective pressures. As a consequence, one possible way to look at the binding properties of simple models of such molecules is to highlight those features which seem to minimize the shortcomings from such a compromise. Correspondingly, these may be identified as (1) the buffering effect from anticooperativity of two binding arms and (2) the reduction of the binding rate arising from reduction of the active site.

Immunoglobulins with two binding lobes encounter more antigens than hypothetical single-domain analogs. Moreover, two binding arms may bind with increased strength on the surface of the same large antigen (as, for example, with proteins on the surfaces of viruses' capsids). However, we have shown that there exists an anticooperative effect due to mutual disturbance between binding domains. We have quantified such negative interference by introducing a simple anticooperativity function. This behaves in a sensible fashion-it decreases (less disturbance) when the two Fabs are farther apart for a given antigen size, or when the size of the antigen decreases for a fixed value of the Fab-Fab separation. Both situations correspond to $\chi=d / 2 R>1$. In contrast, mutual hindrance becomes important when the two Fabs come close to one another, or when the antigen size increases, resulting in a reduction of the encounter rate of as much as $30 \%$. This is the regime $\chi \rightarrow 1$.

The crucial feature of antibodies is their high specificity for antigen binding. Very generally, it is legitimate to speculate that this kind of constraint acted during evolution as an important selective pressure toward the formation of active sites of limited size. However, as a consequence of localization of specific areas, the encounter effectiveness of an antibody is also strongly weakened. In this paper, we have shown that a reduction of the active regions also lessens the effects of mutual disturbance between Fabs. Therefore, if we think of the actual size of the binding sites as the end point of an adaptive evolutionary pathway toward maximization of the encounter effectiveness for a wide class of antigens, the previously mentioned effect might well have acted as an additional selective pressure against other constraints competing toward the enlargement of the specific target zones. For example, one may mention that the cost of 
maintaining two binding arms per antibody in terms of peptide synthesis must be higher than if antibodies were single-domain (possibly fully reactive) agents.

It is interesting to observe that the recovered binding effectiveness due to reduction of anticooperativity is higher the smaller is the active site (Fig. 7). But this is also the regime where the absolute reduction in the binding rate is higher. The negative correlation between these two effects (smaller rate but also smaller anticooperativity) fits well into the picture of antibodies as "flexible" chasers, whereby such molecules are deployed for a wide class of antigen sizes. In fact, it has recently been established that the regions in the Fab tips where the contacts with antigens are made strongly vary in size depending on the size of the antigen (Almagro 2004; MacCallum et al. 1996). Quite generally, antigens may be divided into three categories: proteins (large), peptides (intermediate) and haptens (small). While the contacts involved in binding a protein extend over a wide region on the Fab terminal region, the binding site for a small hapten is buried within a narrow crevice, making its angular aperture extremely small (of the order of a few degrees).

As a final remark, we remind the reader that the configuration of our complex reacting arrangement is fixed (in a sort of handle configuration). Thus, we are not taking into account explicitly dynamical effects associated with the relative motion of the two Fabs, which makes our results valid in the limit of small antigens. The link of the present results with the knowledge accumulated on the dynamics of antibody molecules (Bongini et al. 2004) will be the subject of future research.

Acknowledgements We wish to thank three anonymous referees for their useful comments.

\section{Appendix 1}

In this appendix we illustrate the calculations leading to Eq. 31. Insertion of Eq. 30 into the general expression for the rate (Eq. 23) leads to the following equation:

$$
\begin{aligned}
\kappa & =4 \pi D a \sum_{n=0}^{\infty} \mathrm{e}^{-(2 n+1) \beta_{0}} \\
& \times\left\{\sinh \beta_{0} \mathcal{I}_{3 / 2}(n)+(2 n+1) \tanh \left[\left(n+\frac{1}{2}\right) \beta_{0}\right] \mathcal{I}_{1 / 2}(n)\right\},
\end{aligned}
$$

where

$\mathcal{I}_{p}(n)=\frac{1}{\sqrt{2}} \int_{0}^{\pi} \frac{\sin \alpha P_{n}(\cos \alpha)}{\left(\cos \beta_{0}-\cos \alpha\right)^{p}} \mathrm{~d} \alpha$.

The integral $\mathcal{I}_{1 / 2}(n)$ can be calculated by recalling the identity given in Eq. 27. We obtain

$$
\begin{aligned}
\mathcal{I}_{1 / 2}(n) & =\sqrt{2} \sum_{n=0}^{\infty} \mathrm{e}^{-\left(m+\frac{1}{2}\right) \beta_{0}} I_{n m} \\
& =\frac{2 \sqrt{2}}{2 n+1} \mathrm{e}^{-\left(n+\frac{1}{2}\right) \beta_{0}},
\end{aligned}
$$

where we have made use of the orthonormality of Legendre polynomials,

$I_{n m}=\int_{0}^{\pi} P_{n}(\cos \alpha) P_{m}(\cos \alpha) \sin \alpha \mathrm{d} \alpha=\frac{2}{2 \mathrm{n}+1} \delta_{n, m}$.

The integral $\mathcal{I}_{3 / 2}(n)$ can be easily calculated by noting that

$$
\begin{aligned}
\mathcal{I}_{3 / 2}(n) & =-\left(\frac{2}{\sinh \beta_{0}}\right) \frac{\partial \mathcal{I}_{1 / 2}(n)}{\partial \beta_{0}} \\
& =\frac{2 \sqrt{2}}{\sinh \beta_{0}} \mathrm{e}^{-\left(n+\frac{1}{2}\right) \beta_{0}} .
\end{aligned}
$$

Insertion of Eqs. 54 and 56into Eq. 52 leads to

$\kappa=8 \pi D a \sum_{n=0}^{\infty} \mathrm{e}^{-(2 n+1) \beta_{0}}\left\{1+\tanh \left[\left(n+\frac{1}{2}\right) \beta_{0}\right]\right\}$.

Finally, the expression for the rate constant (Eq. 31) is recovered by substituting into Eq. 57 the definitions of $a$ and $\beta_{0}$ (Eqs. 13, 14).

\section{Appendix 2}

Insertion of Eq. 42 into Eq. 44 leads to the following equation:

$$
\begin{gathered}
G_{0}^{*}=\sum_{n=0}^{\infty}(2 n+1)\left\{X_{n}-\left(q_{n}+X_{n}\right) \tanh \left[\left(n+\frac{1}{2}\right) \beta_{0}\right]\right\} \\
\mathcal{I}_{n}(u)-\sin \beta_{0} \sum_{n=0}^{\infty}\left(q_{n}+X_{n}\right) \mathcal{J}_{n}(u),
\end{gathered}
$$

where

$$
\begin{aligned}
& \mathcal{I}_{n}(u)= \\
& \int_{u}^{\pi} \frac{P_{n}(\cos \alpha) \sin \alpha}{\sqrt{\cos u-\cos \alpha}} \mathrm{d} \alpha . \\
& \mathcal{J}_{n}(u)=\int_{u}^{\pi} \frac{P_{n}(\cos \alpha) \sin \alpha}{\left[\cos \beta_{0}-\cos \alpha\right] \sqrt{\cos u-\cos \alpha}} \mathrm{d} \alpha .
\end{aligned}
$$

These integrals can be solved by introducing the following identity (Sneddon1966):

$\sqrt{2} \sum_{n=0}^{\infty} \cos \left[\left(n+\frac{1}{2}\right) u\right] P_{n}(\cos \alpha)=\frac{\Theta(\alpha-u)}{\sqrt{\cos u-\cos \alpha}}$.

The integral $\mathcal{I}_{n}(u)$ can be easily evaluated using Eq. 60 and recalling the orthonormality condition of Legendre polynomials. We get

$$
\begin{aligned}
\mathcal{I}_{n}(u) & =\sqrt{2} \sum_{n=0}^{\infty} \cos \left[\left(m+\frac{1}{2}\right) u\right] I_{n m} \\
& =\frac{2 \sqrt{2}}{2 n+1} \cos \left[\left(n+\frac{1}{2}\right) u\right] .
\end{aligned}
$$


Insertion of the identity (Eq. 60) into the definition of $\mathcal{J}_{n}(u)$ gives

$\mathcal{J}_{n}(u)=\sqrt{2} \sum_{n=0}^{\infty} \cos \left[\left(m+\frac{1}{2}\right) u\right] \Gamma_{n m}$,

with

$\Gamma_{n m}=\frac{1}{2} \int_{u}^{\pi} \frac{P_{n}(\cos \alpha) P_{m}(\cos \alpha) \sin \alpha}{\cos \beta_{0}-\cos \alpha} \mathrm{d} \alpha$.

The integral in Eq. 63 can be solved by using Eq. 7.224.5 from Gradshteyn and Ryzhik (1980). The result is Eq. 47. Then, insertion of Eq. 58 into Eq. 43 leads to the calculation of two integrals of the type

$\int_{u}^{\pi} \cos \left[\left(p+\frac{1}{2}\right) u\right] \cos \left[\left(q+\frac{1}{2}\right) u\right] \mathrm{d} u=\frac{\pi}{2} S_{p q}$,

with $S_{p q}$ defined as in Eq. 46.

Taking into account expressions Eqs. 58 and 64, it is a simple matter of algebraic rearrangement to put Eq. 43 in the form of the infinite set of Eq. 45.

As a check of consistency, we observe that the case of entirely absorbing Fabs may be easily recovered. In this case one has $S_{m n}=M_{m n}=0$ for all $m, n$, and hence $X_{n}=0$ for all $n$. Consequently, Eq. 40 reduces to Eq. 29, and the solution (Eq. 30) is recovered along with the rate constant (Eq. 31) (see also Eq. 48). On the other hand, the solution to the Laplace problem must vanish in the limit $\alpha_{0} \rightarrow 0$, where the two Fabs become entirely reflecting. In this case one has $S_{m n} \rightarrow \delta_{m n}$, and hence the problem reduces to solving the following infinite homogeneous system in the variables $X_{n}+q_{n}$ (see Eq. 40),

$\sum_{m=0}^{\infty} \Xi_{n m}\left(X_{m}+q_{m}\right)=\sum_{m=0}^{\infty}\left(\delta_{n m}+M_{n m}\right)\left(X_{m}+q_{m}\right)=0$,

whose only solution is the trivial one $X_{n}+q_{n}=0 \forall n$, being $\operatorname{det}(\Xi) \neq 0$ at each order of truncation (which can easily be proved numerically to be the case). This proves

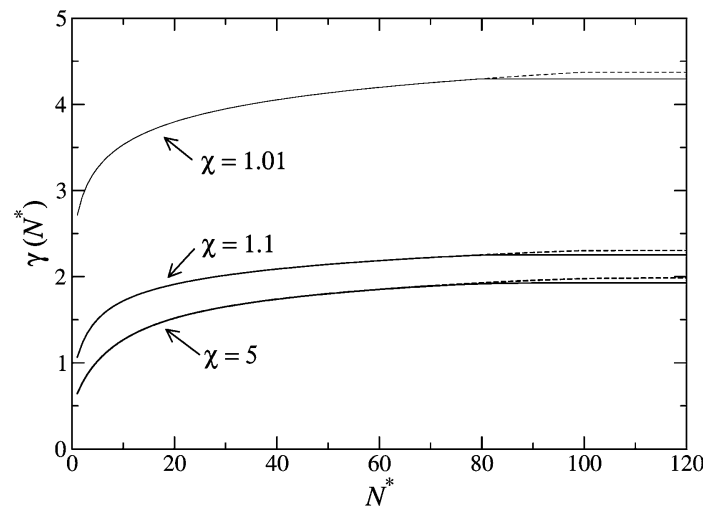

Fig. $9 \gamma\left(N^{*}\right)$. Solid lines, $N_{t}=80$ Dashed lines, $N_{t}=100$ that the solution to the Laplace problem is indeed the trivial one, and that the rate constant vanishes (see also Eq. 48).

\section{Appendix 3}

In this appendix we discuss in some detail the convergence properties associated with truncation of the infinite set of equations (Eq. 45).

If we indicate with $N_{t}\left(\theta_{0}, \chi\right)$ the number of equations retained in the infinite system (Eq. 45) for a given choice of the parameters $\left(\theta_{0}, \chi\right)$ within the intervals of interest, we find that a relative error $\delta X_{n} / X_{n}<0.1 \% \forall n$ may be obtained by keeping in the worst case up to order $N_{t}=100$ equations. In the calculation of the norm of $\mathbf{M}$, the sum in the definition (Eq. 46) has been truncated at a fixed number of terms $N^{*}$, independent of $N$. To understand whether that is legitimate, let us consider that term in more detail. Since $\left|S_{m n}\right|<1 \forall m$,n, we have

$$
\begin{aligned}
\left|\sqrt{\chi^{2}-1} \sum_{m=0}^{N_{t}} \sum_{k=0}^{N *} \Gamma_{m k} S_{k n}\right| & <\sqrt{\chi^{2}-1} \sum_{m=0}^{N_{t}}\left|\sum_{k=0}^{N *} \Gamma_{m k}\right| \\
& <\sqrt{\chi^{2}-1} \sum_{m=0}^{N_{t}}\left|\sum_{k=0}^{N *} \Gamma_{m k}\right| \underline{\underline{\operatorname{def} \gamma}}(N *) .
\end{aligned}
$$

The quantity $\gamma\left(N^{*}\right)$ is plotted in Fig. 9 for three values of $\chi$, with two choices of the truncation order $N_{t}$ each. It is very clear that such an indicator converges rapidly for all values of $\chi$. The plot confirms that the correct value of $N^{*}$ in order to assure convergence increases with the order of truncation $N_{t}$. In particular, we have $N^{*} \approx N_{t}$. Hence, the greater precision we require in the numerical solution for the coefficients $X_{n}$, the greater must be $N_{t}$, and consequently the greater $N^{*}$. Operatively, we can thus safely require the specified accuracy to give the rules for both truncations.

\section{References}

Almagro JC (2004) J Mol Rec 17:132

Barzykin AV, Shushin AI (2001) Biophys J 80:2062

Bongini L, Fanelli D, Piazza F, De Los Rios P, Sandin S, Skoglund U (2004) PNAS 101:6466

Gradshteyn IS, Ryzhik IM (1980) table of integrals, series and products. Academic, London

Kitano H, Iwai S, Okubo T, Ise N (1987) J Am Chem Soc 109:7608

Levison SA, Jancsi AN, Dandliker WB (1968) Biochem Biophys Res Commun 33:942

Loset GA, Roux KH, Zhu P, Michaelsen TE, Sandle I (2004) J Imm 172:2925

MacCallum RM, Martin ACR, Thornton JM (1996) J Mol Biol 262:732

Morse PM, Feshbach H (1953) Methods of theoretical physics. Mc Graw-Hill, New York

Noble RW, Reichlin M, Gibson QH (1969) J Biol Chem 244:2403

Potter MJ, Luty B, Zhou HX, McCammon JA (1996) J Phys Chem 100(12):5149 
Sandin S, Öfverstedt L-G, Wikström A-C, Wrange Ö, Skoglund U (2004) Structure 12(3):409

Smoluchowski MV (1916) Phys Z 17:557

Smoluchowski MV (1917) Z Phys Chem 92:129

Sneddon IN (1966) Mixed boundary value problems in potential theory. North-Holland, Amsterdam
Song YH, Zhang YJ, Shen TY, Bajaj CL, McCammon A, Baker NA (2004) Biophys J 86(4):2017

Tanimoto S, Kitano H (1993) Langmuir 9:1315

Traytak SD (1995) Chem Phys 192:1

Voet D, Voet JG (1995) Biochemistry. Wiley, New York 